ISSN 1997-5902

\title{
La crise de la filière cotonnière et sécurité alimentaire au Nord Cameroun
}

\author{
Denis Pompidou FOLEFACK ${ }^{1 *}$, Jeshma NTSOU BAKWOWI2, Patrice COKOU KPADE ${ }^{3}$ \\ ${ }^{1}$ Ministère de la Recherche Scientifique et de l'Innovation, B.P. 1457 Yaoundé, Cameroun \\ 2 Centre National de l'Education, Yaoundé, Cameroun \\ ${ }^{3}$ Université d'Agriculture de Ketou, Kétou, Benin \\ *auteur correspondant : Email : dfolefack@yahoo.fr
}

Original submitted in on 12th November 2013. Published online at www.m.elewa.org on 31st March 2014. http://dx.doi.org/10.4314/jab.v75i1.8

\section{RÉSUMÉ}

Contexte et objectif : Le coton a longtemps contribué à la sécurité alimentaire, du fait de son rôle historique joué sur la productivité agricole. Cette étude à pour objectif d'apporter des éléments de contribution aux débats actuels sur les effets de la crise de la filière cotonnière sur la sécurité alimentaire.

Méthodologie et Résultats : Depuis les années 2000, les crises successives du marché mondial ont affecté fortement la filière cotonnière. Les céréales ont été les cultures les plus touchés par la crise cotonnière, notamment dans la fourniture des intrants (engrais). Face au contexte défavorable de la culture du coton, les exploitations agricoles diversifient leurs activités, en réorientant leurs activités vers les cultures céréalières, mais, force est de constater que les superficies cultivées restent très faibles, les revenus sont limités et en baisse. Dans ce contexte, la sécurité alimentaire reste très précaire et des risques de famines sont permanents, ceci est dû au fait que les conditions d'accès aux intrants sont plus complexes et les coûts de plus.

Conclusion et application de résultats: Cette situation a des effets directs sur les rendements, la production et l'offre en produits céréaliers dans les marchés. On note une difficile adaptation du système de production céréalière locale. Des actions interventions fortes doivent faites pour renforcer la sécurité alimentaire dans cette région.

Mots clés : Crise cotonnière ; sécurité alimentaire ; filière ; céréales; Nord Cameroun.

\section{Cotton crisis and food security in Northern Cameroon \\ Abstract}

Context and objective: Cotton has contributed to food security for longtime, because of its historic role in agricultural productivity. This study aims to provide some contribution to current debates on the effects of the crisis in the cotton industry on food security.

Methodology and Results: Since 2000, the successive crises of the world market have strongly affected the cotton industry. Cereals have been the most affected by the cotton crisis, particularly in the supply of inputs (fertilizer). Faced with the unfavorable the cotton crop, farmers are diversifying their activities by shifting to cereals crops, but it is clear that the cultivated areas are still very small, the incomes are limited and decreasing. In this context, food security remains precarious and the risks of famine are permanent, this is due to the fact that the conditions for access to more complex inputs have become more expensive. 
Conclusion and application of results: This situation directly affects the yield, production and supply of cereal products in the markets. There is a difficult adaptation of the local cereals farming system and Strong actions and interventions are needed to enhance food security in the region.

Keywords: cotton crisis, food security, industry, cereals, North Cameroon.

\section{INTRODUCTION}

La sécurité alimentaire se définit comme l'accès permanent de tous aux denrées alimentaires nécessaires pour mener une vie saine et active (Banque Mondiale, 1986). Par cette définition, la réalisation de la sécurité alimentaire reste un défi majeur à relever en Afrique sub-saharienne. En effet, 212 millions de personnes, soit 30\% de la population totale, souffrent de la faim. Ces chiffres démontrent à suffisance l'ampleur du problème de sécurité alimentaire dont les conséquences à terme sont la dégradation de la qualité de bien être social pour la grande majorité des populations (FAO, 2008 ; Dembélé et Staatz, 2010). La question d'insécurité alimentaire constitue encore une des contraintes majeures du développement de nombreux pays d'Afrique Subsaharienne. On estime que plus d'un tiers des populations de ces pays ne mangent suffisamment pas leur faim pour mener une vie saine et active, et ce problème est encore plus marqué chez les jeunes enfants et autres groupes vulnérables (FAO, $2006 ; 2008$; Mérino, 2009). Au Cameroun, près de $25 \%$ de la population camerounaise serait touchée par l'insécurité alimentaire avant le début de la crise alimentaire mondiale (MINEPAT, 2008). La hausse de l'indice des prix à la consommation des ménages et la forte reprise des importations alimentaires depuis 1996 semblent confirmer la faible croissance de certaines productions alimentaires (céréales, poisson, produits animaux, etc.) par rapport à la demande. Au Nord Cameroun, le problème d'insécurité alimentaire est chronique et récurrent. Mais, la culture cotonnière a joué un rôle primordial dans la transformation agricole et par ricochet sur la sécurité alimentaire, du fait de la forte corrélation entre la hausse de la production cotonnière et la production céréalière (SODECOTON, 1999 ; Deveze, 2006 ; Folefack, 2009 ; PAM ; 2011). Cependant, le déficit céréalier s'est accru ces dernières années par une combinaison de l'augmentation de la population, et de la variabilité inter annuelle des productions vivrières dues aux aléas climatiques, et plus récemment de la crise cotonnière. En effet, depuis les années 1990, les crises successives du marché mondial ont affecté fortement la filière cotonnière au Cameroun. La baisse du cours mondial du coton a été amplifiée par la dépréciation du dollar américain face à l'euro et au FCFA qui lui est lié (Berti et al., 2006 ; Tschirley et al., 2009). II y a aussi d'autres facteurs plus structurels et plus spécifiques au pays comme le mode d'organisation et de fonctionnement de la filière (Gafsi et Mbetid-Bessane, 2001 ; MbetidBessane et al., 2003). Entre 2001 et 2007, la régression de la filière cotonnière se décline en baisse des superficies $(-40 \%)$, de la production ($40 \%)$, des rendements $(-28 \%)$ et des recettes d'exportations (-35\%) (Folefack, 2010). Selon les experts, les événements climatiques extrêmes risquent également de s'aggraver et de se multiplier, renforçant la volatilité de la production et des prix alimentaires. Ce sont des conditions climatiques défavorables qui ont contribué à la crise alimentaire de 2008 tandis que les sécheresses, les précipitations excessives et les inondations ont affecté les récoltes des grands producteurs céréaliers et alimenté une augmentation des prix tout au long de l'année 2010. L'indice des prix alimentaires de la Banque mondiale a augmenté de pratiquement $30 \%$ l'année dernière et, selon le Food Price Watch, cette flambée des prix aurait plongé près de 44 millions de personnes dans l'extrême pauvreté.

Selon le PAM (2011), au Cameroun, les ménages en insécurité alimentaire recourent à des stratégies de survie pour faire face au manque d'argent et de nourriture, mais la fréquence de ce recours reste cependant limitée. Les stratégies utilisées sont entre autres : la diversification des activités agricoles dans l'exploitation (avec la prédominance des céréales) ; la consommation 
des aliments moins chers, réduire la quantité de nourriture consommée et réduire le nombre de repas. Face à cette situation problématique sur la sécurité alimentaire, et comme l'a signalée la Banque mondiale, «nous devons agir ensemble pour relancer la production et la productivité agricoles mais aussi pour améliorer les réseaux de distribution, afin d'assurer la sécurité alimentaire et permettre aux populations de se procurer la nourriture nécessaire $॥$.

\section{MATERIELS ET METHODES}

Zone d'étude et collecte des données : Entre 2005 et 2009 , les études menées sur les terroirs de référence du Pôle de recherche appliquée au développement des savanes d'Afrique centrale (PRASAC) et le projet d'Appui de Recherche appliquée au Développement des Savanes d'Afrique Centrale (ARDESAC), répartis sur l'ensemble de la zone cotonnière du Nord Cameroun, couvrant, les régions du Nord et de l'Extrême Nord, ont permis de caractériser les exploitations agricoles familiales. Ces régions ont été choisies pour cette analyse, car, le problème d'insécurité alimentaire ${ }^{1}$ affecte sérieusement l'activité agricole, les mois de disette coïncidant généralement avec la période d'intenses travaux champêtres. Pendant longtemps, les aléas climatiques ont été mis en avant comme cause explicative de la famine. Une analyse documentaire importante a été effectuée et les données recherchées concernent les aspects suivants: production céréalière, crise cotonnière, diversification des activités dans les exploitations, stockage des céréales et des contraintes.

Concept de sécurité et d'insécurité alimentaire : Pour mieux orienter nos analyses, il est utiliser tout d'abord d'apporter des éclairages sur les concepts clés utiliser dans cet article. La notion d'insécurité alimentaire a considérablement évolué depuis le Sommet mondial de l'alimentation qui s'est tenu Rome en 1974. Au début, l'insécurité alimentaire était considérée comme une défaillance (temporaire) dans les approvisionnements alimentaires au niveau global, notamment au niveau de la production nationale, causée par une catastrophe naturelle comme la sécheresse ou les inondations. Cette évolution a mené, depuis les années 1970, à de nouvelles définitions de

\footnotetext{
${ }^{1}$ Selon le PAM,

l'insécurité alimentaire sévère touche surtout l'Ext rême Nord (4,1\% des ménages) et le Nord (3,7\%).
}

Cette étude est une contribution aux débats actuels sur les effets de la crise de la filière cotonnière sur la sécurité alimentaire en zone cotonnière. Ce travail s'articule autour de plusieurs parties, à savoir : L'état de la filière cotonnière en crise au Nord Cameroun ; les effets de la crise cotonnière sur la sécurité alimentaire et les actions à mener pour renforcer la sécurité alimentaire.

la notion de sécurité alimentaire. La Banque Mondiale (1986) l'a définie en ces termes : accès par tous et tout moment aux denrées alimentaires nécessaires pour mener une vie saine et active. Selon cette définition, les problèmes d'accès aux denrées suffisantes sont désignés sous le terme d'insécurité alimentaire. On fait généralement le distinguo entre l'insécurité alimentaire chronique et l'insécurité alimentaire passagère, c'est-àdire la défaillance permanente par rapport à la défaillance temporaire des modes d'accès aux denrées alimentaires nécessaires. La vulnérabilité à l'insécurité alimentaire peut être définie comme le risque encouru ou la fragilité laquelle on s'expose en cas de perte soudaine de revenus ou de non accès la nourriture, ce qui peut précipiter une crise alimentaire en l'absence d'interventions compensatoires. En général, cette fragilité se réfère aux risques de sous-alimentation, qu'ils soient de nature biologique, socioéconomique, politique ou environnementale. On distingue donc plusieurs catégories de groupes vulnérables. Les groupes biologiquement vulnérables correspondent aux personnes qui, en raison de leur état physiologique, courent un très haut risque de souffrir de malnutrition. On citera par exemple les femmes enceintes ou allaitant, et les très jeunes enfants de 6 à 36 mois dans leur période de sevrage. Les groupes socio économiquement vulnérables comprennent les personnes qui, sujettes à la pauvreté en raison de la médiocrité de leur condition socioéconomique, n'ont pas suffisamment accès à une nourriture de qualité. On trouve dans ce groupe des paysans démunis, et les pauvres des zones urbaines dont la survie dépend d'activités relevant du secteur informel. Les groupes politiquement vulnérables sont ceux qui, pour raison de guerres ou autres conflits sociaux, n'ont pas suffisamment accès la nourriture. Enfin, les groupes écologiquement vulnérables sont ceux qui vivent dans des espaces marginaux caractérisés par un manque de 
ressources naturelles ou enclins aux catastrophes naturelles comme la sécheresse ou les inondations. II faut cependant rappeler que ces différents types de vulnérabilité tendent à se chevaucher.

\section{RESULTATS}

Effets de la crise cotonnière sur la production des céréales au Nord Cameroun : Comme dans les autres pays d'Afrique francophone producteurs de coton, la baisse des prix internationaux du coton et la montée de l'Euro par rapport au dollar ont eu un impact considérable sur les prix du coton exprimés en FCFA. Cela s'est traduit par le déclin de la filière cotonnière (voir indicateur tableau 1) et une fragilisation de la position financière de la société cotonnière. Celle-ci a diminué le montant des crédits intrants fournis aux producteurs. Ceux-ci ont alors diminué leurs achats d'intrants, dont les prix ont par ailleurs augmenté, entraînant une baisse des rendements de $28 \%$ entre 2001 et 2007. Cette baisse des rendements, associée à la baisse des prix du coton-graine payés aux producteurs $(10 \%)$, diminue leurs ressources financières. Ceci accroît leur risque de se voir refuser l'accès aux crédits intrants lors de la campagne suivante, ce qui précipite la chute des rendements cotonniers (Folefack, 2009 ; 2010 ; Fok, 2010).

Tableau 1 : Évolution des indicateurs de production du coton au Cameroun

\begin{tabular}{lcccccc}
\hline \multirow{2}{*}{ Campagne } & Surface & Production & \multirow{2}{*}{$\begin{array}{c}\text { Rendement } \\
\text { (Kg/ha) }\end{array}$} & $\begin{array}{c}\text { Prix moyen } \\
\text { d'achat (FCFA) }\end{array}$ & NPK & Urée \\
\hline $2000 / 2001$ & 193627 & 230932 & 1163 & 193 & 11500 & 10000 \\
$2001 / 2002$ & 201576 & 246070 & 1221 & 183 & 12000 & 10500 \\
$2002 / 2003$ & 181810 & 233803 & 1286 & 179 & 11500 & 10000 \\
$2003 / 2004$ & 208204 & 242819 & 1166 & 185 & 11500 & 10000 \\
$2004 / 2005$ & 215027 & 304053 & 1414 & 189 & 12500 & 11000 \\
$2005 / 2006$ & 231993 & 220839 & 952 & 172 & 13500 & 11500 \\
$2006 / 2007$ & 203023 & 183710 & 905 & 172 & 15500 & 15300 \\
$2007 / 2008$ & 137869 & 145000 & 1020 & 185 & 14500 & 14500 \\
$2008 / 2009$ & 147433 & 146794 & 996 & 183 & 18800 & 18800 \\
\hline
\end{tabular}

NB : Un sac $=50 \mathrm{~kg}$

Cependant, d'autres facteurs plus structurels sont à l'origine de la crise, notamment : le mode d'organisation et de fonctionnement, la crise de confiance entre les producteurs et les sociétés cotonnières en liaison avec des erreurs de gestion (Badiane et al., 2002 ; Mbetid-Bessane et al., 2006). La conjonction de facteurs internationaux, régionaux et locaux a entraîné la filière cotonnière camerounaise dans un cercle vicieux, avec comme résultats la baisse de la production de coton ( $40 \%$ ) et des céréales avec la montée de la pauvreté dans les villages. En effet, avec la crise cotonnière, l'on note une diminution des revenus des $9 \%$ malgré la diversification et une réorientation des activités vers les vivriers (Folefack, 2009). Par ailleurs, la montée de la pauvreté dans les groupements incite les producteurs à revendre une partie de leurs intrants, normalement destinés à la culture cotonnière, ou à les appliquer sur des cultures vivrières, aux dépens de la culture du coton. II faut noter que la filière coton est quasiment la seule source d'intrants et de crédits pour les cultivateurs, d'où des détournements importants au profit des autres cultures. La baisse de production de coton affecte également la production de tourteau de graines de coton, au détriment des élevages et de la culture attelée. Par ailleurs, la montée du climat d'insécurité dans certaines zones de production du coton augmente les risques de vols. Enfin, le manque de terres à défricher implique que la production du coton doit se faire sur des sols déjà cultivés depuis de nombreuses années et souvent appauvris. Suite aux programmes d'ajustement structurel engagés sous l'impulsion des institutions des bailleurs de fonds, l'Etat s'est désengagé et les subventions aux intrants ont été suspendues. Cette 
réforme a entraîné la hausse significative des prix des intrants. Prenons le cas de l'engrais qui est le facteur de production ayant un coût très élevé. Le prix du sac d'engrais NPK (50 kg) (22-10-15 et 15-20-15) et de l'Urée sont passés de 11500 FCFA en 2000/2001 à 17 000 FCFA en 2008/2009, soit une augmentation du prix de près de $48 \%$. En plus, depuis la campagne 2005/2006, les modalités de session des intrants vivriers par la SODECOTON ont été modifiées avec la limitation des commandes en fonction des surfaces de coton réalisées. II a été retenu un maximum de 2 sacs d'engrais vivriers (NPK ou Urée) pour un hectare de coton, avec la condition d'avance soit par le producteur de $50 \%$ du prix d'achat ou un achat au comptant. Bien que des efforts de modernisation des principales cultures vivrières (sorgho, mil, maïs, arachide et niébé), mais, les difficultés de la filière se sont transportées au niveau de ces cultures, auxquelles elle assurait jusqu'à présent la fourniture des intrants. Les producteurs pauvres n'ont pas les moyens pour payer les avances ou au comptant les intrants vivriers, ce qui limitent l'utilisation des intrants vivriers dans la zone. Par ailleurs, l'absence de toute organisation pour ces filières ne permet pas que la situation s'améliore. En plus, la pauvreté ambiante, les producteurs sont obligés d'écouler leurs céréales au moment de la récolte, quand les prix sont au plus bas. En effet, selon Institut National de la Statistique (2008), la situation du Nord Cameroun est d'autant plus préoccupante qu'elle perdure et semble s'aggraver au fil du temps. Du reste, c'est dans l'Extrême Nord et le Nord que le gap est le plus important entre le niveau de la consommation moyenne des pauvres et le seuil de pauvreté situé près de $60 \%$. Ce mauvais résultat semble provenir des obstacles d'ordre conjoncturel et structurel. Sur le plan conjoncturel, il y a eu des inondations, des invasions de pachydermes et d'oiseaux granivores dans cette partie du pays en 2007. Ces calamités ont eu pour conséquence des pertes en biens et en récoltes qui ont affecté négativement les populations. Sur le plan structurel, les pratiques agricoles archaïques et le déficit chronique pluviométrique ont pour conséquence une production agricole insuffisante pour que les populations soient à l'abri de l'insécurité alimentaire. De plus, les paysans sont victimes de l'absence de techniques de conservation qui les pousse à vendre l'essentiel de leurs récoltes au moment de la moisson et d'emprunter à des taux d'intérêt élevés pour acheter en période de soudure. En outre, suite à la crise et la politique cotonnière actuelle, près de 100000 producteurs ont abandonnés ou été exclus de la culture cotonnière au cours de ces dernières années (à cause des impayés dans les cercles de caution solidaire et groupements). Avec cette proportion importante des producteurs ne cultivant pas de coton, c'est un manque à gagner important des engrais pour la production des vivriers (céréales). La seule source formelle d'approvisionnement en intrants reste I'OPCCGIE/SODECOTON (filière coton pas encore libéralisée), des tentatives privées ont été faites par le passé, mais se sont toutes soldées par des échecs. Avec le niveau de revenus faibles et la pauvreté ambiante, les producteurs n'ont pas les moyens financiers pour acquérir les intrants au comptant. Une infirme partie des producteurs vivriers achètent des intrants pour leurs vivriers aux producteurs de coton qui bradent leurs intrants. Le manque de crédits intrants et d'engrais a pour conséquence directe, la baisse inéluctable des rendements et de la production céréalière. Cette baisse de la production réduit ainsi l'offre céréalière dans les marchés et par conséquent, une instabilité et une augmentation des prix sur le marché comme le montre la figure 1. Cette instabilité des prix des céréales locales révèle une situation de risque pour les producteurs. Elle crée un facteur limitant pour des innovations techniques qui augmentent la dépendance au marché c'est à dire qui induisent des coûts monétaires : acquisition de semences améliorées ou d'intrants chimiques. La régulation du prix des marchés du mil ou du moins la diminution des sources d'instabilité est potentiellement un axe d'intervention qui renforcerait la capacité d'innovation des filières locales (Temple et al, 2009). 


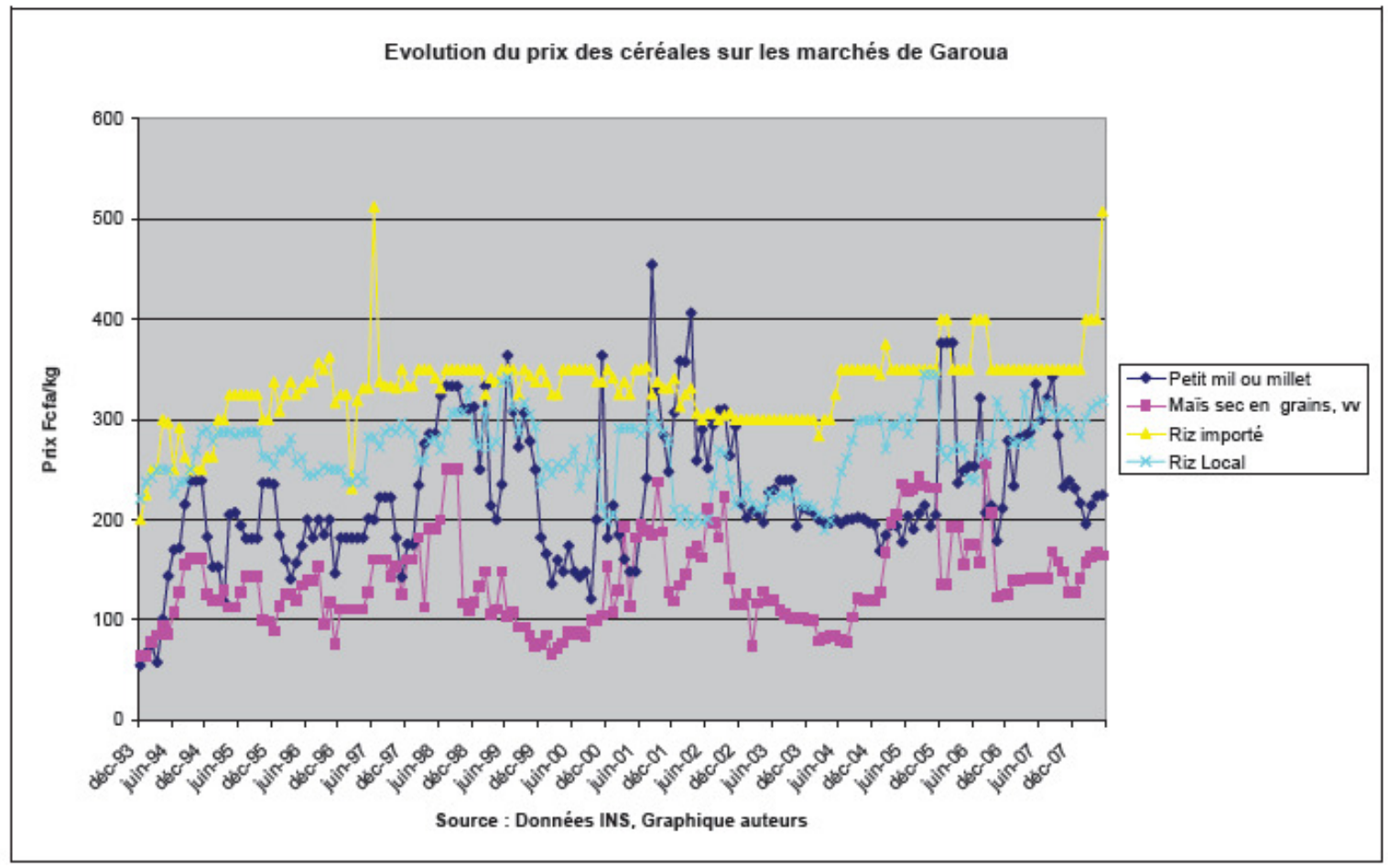

Source : Temple et al (2009)

Figure 1 : Evolution du prix des céréales sur les marchés de Garoua (2003-2007).

En effet, d'après Temple et al (2009), le NordCameroun traverse en effet régulièrement des périodes d'insécurité alimentaire qui se traduisent par un appel aux importations ou une mobilisation de l'aide alimentaire mondiale. Ne pouvant spécifier sur les données mobilisables des importations alimentaires régionales du Nord-Cameroun, nous posons pour hypothèse que les indicateurs nationaux sont représentatifs de la situation observée dans les villes $\mathrm{du}$ nord. Cette hypothèse est fondée sur deux indicateurs : l'importance des céréales dans le modèle alimentaire du Nord-Cameroun par rapport au sud et le rythme de croissance des populations urbaines plus élevé dans le nord. Le rythme continu d'augmentation des importations de céréales en volumes au niveau national (plus de 100000 tonnes) se concentre sur le blé et le riz. En calculant un indicateur de dépendance alimentaire par le ratio quantité importée/quantité produite, nous constatons l'accroissement exponentiel de ce ratio qui est passé de $20 \%$ à $40 \%$ en 8 ans. De manière complémentaire on note une augmentation significative de la quantité de céréales importées par habitant. Comme la ration calorique a globalement stagné, voire diminué, au Cameroun, l'accroissement des importations par habitant confirme une substitution des céréales importées aux vivriers locaux et aux céréales locales.

Diversification des activités dans les exploitations agricoles en faveur des vivriers : La diversification des activités dans les exploitations apparaît comme un choix des agriculteurs pour gérer les risques : la culture cotonnière, les cultures vivrières, les élevages et les activités non agricoles forment la base du système de production (Folefack, 2009 ; Mana et al., 2009). La crise cotonnière a entrainé une diminution importante des superficies cultivées en coton au profit des vivriers, même si les parcelles restent petites et les productions faibles, et des modifications dans les assolements des exploitations agricoles. Les producteurs cherchent en premier lieu à renforcer la sécurité alimentaire. Les exploitations développent diverses stratégies d'adaptation. A titre d'illustration, l'analyse nous montre quatre catégories d'exploitations agricoles : Ainsi, la première catégorie des producteurs dans leur majorité baisse leurs superficies de coton d'environ $76 \%$, passant de 2,4 hectares avant la crise à 0,58 hectare avec la crise au profit des céréales et des légumineuses : maïs, 2 hectares; arachide, 1,8 hectare ; sorgho, 1 hectare ; muskwari, 1,26 hectare et niébé, 0,36 hectare. La deuxième catégorie des producteurs 
augmente la part du coton dans l'assolement de près de $10 \%$, passant de 0,97 hectare avant la crise à 1,07 hectare, et simultanément les superficies de certains vivriers : maïs, 1,37 hectare; arachide, 1,08 hectare ; sorgho, 0,82 hectare ; muskwari, 1 hectare et niébé, 0,50 hectare. La troisième catégorie abonne le coton soit volontairement ou sont exclu augmentent la part des vivriers : maïs, 0,42 hectare; arachide, 0,50 hectare ; sorgho, 0,82 hectare ; muskwari, 1,75 hectare et niébé, 0,15 hectare. Et enfin, la quatrième catégorie garde les superficies des différentes spéculations stables : coton, 1,56 hectare ; maïs, 1,94 hectares; arachide, 1.5 hectare ; sorgho, 0,5 hectare ; muskwari, 1 hectare et niébé, 0,38 hectare. Malgré les réajustements des exploitations, la précarité de la sécurité alimentaire reste et les revenus des producteurs sont en baisse au cours de ces dernières années, passant de 270000 FCFA en 2000 à 246565 FCFA en 2007, soit une baisse d'environ 9\%. Dans ce revenu moyen, la part du coton à nettement baissé passant de $60 \%$ à $11 \%$, soit 27900 FCFA ; la part des vivriers est de $75085 \mathrm{FCFA}$, soit $30 \%$; l'élevage 32 335 FCFA, soit $13 \%$ et les activités extra agricole, 111 245 FCFA, soit $45 \%$. Les producteurs de la zone cotonnière estiment que les revenus de leurs différentes activités ne permettent pas de satisfaire des charges importantes du ménage dans un environnement économique marqué par des fortes inflations. En outre, avec ces mutations observées dans les exploitations, les producteurs atteignent difficilement les objectifs des exploitations, qui sont d'assurer la satisfaction des besoins alimentaires et l'accroissement des revenus des exploitations. II est à rappeler qu'au Nord Cameroun, selon Temple et al (2009) les coefficients budgétaires (\% de la dépense alimentaire pour un produit) montrent l'orientation céréalière du modèle alimentaire du Nord-Cameroun ( $90 \%$ des dépenses alimentaires portent sur les céréales et $10 \%$ pour les tubercules et féculents). Les racines, tubercules et féculents (manioc, patate, igname, plantain) restent des produits de consommation marginale pour les populations majoritairement originaires du Nord-Cameroun.

Difficile adaptation de systèmes de production céréaliers locaux : L'inertie technologique constatée sur les céréales locales selon la grille d'analyse mobilisée pourrait s'expliquer par deux facteurs. En premier lieu l'instabilité actuelle du marché local étant extrêmement forte en comparaison avec d'autres céréales (le maïs en l'occurrence), cette instabilité engendre une aversion à l'investissement dans des innovations de rupture (utilisation d'intrants) par rapport aux formes d'intensification écologique que produisent les sociétés locales. Les mils sorghos ne bénéficient pas des mêmes niveaux d'investissement en termes de recherche agronomique au public et privé. Enfin, ils ont moins bénéficié des effets d'entraînements (par comparaison avec le maïs et le riz) induit par les firmes cotonnières notamment dans les conditions de sécurisation des approvisionnements en semences. Ainsi on note une certaine inégalité sur les conditions d'accès aux semences entre les différentes céréales. Entre 2005 et 2007, la production régionale de semences certifiées a été de 544 tonnes en sorgho, 912 tonnes en riz et 3431 tonnes en maïs. L'offre en semences améliorées des céréales locales reste donc faible pour une superficie moyenne annuelle en mil/sorgho de 240000 ha (Fofiri et al., 2008). Face à l'insécurité alimentaire, l'action publique est généralement orientée dans trois directions : la lutte contre les grands fléaux, la modernisation de l'agriculture et l'assistance vivrière. Si la lutte contre les fléaux s'appuie sur les services phytosanitaires dont les moyens sont souvent insuffisants ou tardivement déployés, la modernisation de l'agriculture reste un discours parfois incantatoire sur l'amélioration de la productivité. L'assistance alimentaire est par contre l'action la plus visible sur le terrain, sous forme de mise en place de structures publiques de stockage vivrier dans les zones à risque. L'office céréalier créé en 1975 est la principale structure publique fonctionnant dans ce sens, même si son fonctionnement souffre régulièrement d'un certain nombre de handicaps. En effet, la principale source de cette structure est la subvention de l'Etat qui connaît des irrégularités, compromettant ainsi son bon fonctionnement. Par ailleurs, les fréquentes réformes que connaît la structure contribuent quelquefois à réduire ses marges de manœuvre (Fofiri et al., 2008 ; Temple et al, 2009).

D'autre part, le déploiement territorial des activités du Programme Alimentaire Mondiale (PAM) dans la région du Nord œuvre dans le même sens. II convient toutefois de relever les limites fonctionnelles de ces structures en raison de problèmes de gouvernance, notamment la forte implication des autorités administratives et traditionnelles dans la gestion des aides PAM, les détournements réguliers de stocks destinés à l'appui à la scolarisation des jeunes ruraux par les directeurs d'école. L'absence de bonne gouvernance constitue de nos jours le principal handicap à une meilleure redistribution des aides PAM, dans l'optique d'une réduction de l'insécurité 
alimentaire régionale (Temple et al, 2009). L'action des ONG, quant à elle, contribue dans une certaine mesure à limiter les conséquences de la mauvaise gouvernance des structures publiques de stockage, par des opérations innovantes impliquant et responsabilisant les producteurs, même si le « droit de regard » dont jouissent ces organismes au nom du respect de la démocratie et de l'éthique, est de plus en plus le point de friction avec l'Etat, compromettant ainsi la bonne exécution de nombreux projets de développement rural. II en est ainsi du développement des systèmes de stockage villageois sous forme de greniers communautaires et de groupements de stockeurs pilotés par les organisations paysannes et appuyés par des systèmes d'aide à la gestion. Sans être exhaustives, les contraintes à la production qui persistent dans la région, interrogent sur l'impact réel des interventions diverses financées par la communauté des bailleurs de fonds internationaux et de l'Etat, sur l'amélioration des systèmes de production régionaux. Car, l'impact se mesure par des changements techniques dans la pratique des agriculteurs et l'amélioration des revenus des populations à la suite de la diffusion et de l'adoption des innovations agricoles.

\section{Actions à mener pour renforcer la sécurité alimentaire}

Favoriser le stockage villageois des céréales : En milieu rural sahélien, les céréales locales, essentiellement le mil et le sorgho, qui constituent la base de l'alimentation, sont récoltées à l'issue de l'unique saison des pluies (aux alentours de juin septembre). Elles sont stockées dans des greniers familiaux traditionnels afin d'assurer l'alimentation jusqu'à la récolte de l'année suivante. Mais dans les zones déficitaires, même ces stocks sont insuffisants. En effet, l'une des options stratégiques susceptibles de renforcer la sécurité alimentaire en général et de stabiliser les prix des produits de base en particulier, consiste à créer des stockages de céréales, opération organisée au niveau du village et gérée par la collectivité qui achète, engrange et vend les céréales. Le rétablissement de ces banques de céréales répond généralement à une logique parfaitement justifiée. Au Nord Cameroun, la récolte se fait généralement entre septembre et novembre et en février et mars pour le cas du muskwaari, ce qui demande aux paysans de stocker au moment des récoltes pour aller jusqu'à la suivante, ce qui a aussi une incidence sur les prix qui peuvent varier fortement sur l'année. Pour améliorer la sécurité alimentaire des ménages, il faut donc les aider à stocker les céréales et trouver les moyens de réguler les prix. D'où les actions de stockage villageois, mais aussi le rôle de l'Office céréalier pour limiter la spéculation. Le stockage comme outil de régulation des crises alimentaires n'est pas une recommandation nouvelle. II est promu, avec plus ou moins de bonheur, depuis la période coloniale. Certaines expériences de stockage au village, remontent aux années 60 . L'Office céréalier, instrument de la politique nationale de sécurité alimentaire, œuvre depuis longtemps pour la mise en place de stocks vivriers dans les régions à risques. D'anciennes malversations financières ont nui à la crédibilité de cet organisme, mais le dispositif d'aide d'urgence reste indispensable en cas de famine exceptionnelle. Des interventions plus localisées se sont orientées vers un système de stockage de proximité géré par les producteurs. II s'agit essentiellement de banques de céréales suscitées soit par les groupements cotonniers. Leurs promoteurs insistent sur le caractère social de ce système de stockage, basé essentiellement sur des achats de céréales en période de récolte et destiné en priorité aux plus indigents en période de "soudure " (Abdourahmane et al., 2002).

Ainsi, pour renforcer la sécurité alimentaire des efforts sont actuellement faites par l'OPCC-GIE et autres ONG en stockant les céréales dans les magasins et en assurant une bonne gestion des stocks durant les périodes de soudure. A titre d'illustration, au cours de la campagne 2006/2007, les actions entreprises sont poursuivis pour soutenir les producteurs céréaliers a fin de les aider à mieux gérer les stocks. Pour cette campagne, plus de 120 millions ont été distribués à plus de 12000 producteurs stockeurs de céréales ce qui a permis de mettre en réserve dans les magasins près de 30000 sacs de sorgho et de maïs. Le service que l'OPCC-GIE a mis en place avec le réseau des animateurs a également apporté son appui à d'autres types de stockage : dépôt simple des sacs de membres sans avance (1 680 sacs stockés) et dépôt avec avance des groupements (8000 sacs pour 40 millions d'avances) (SODECOTON, 2008). Pour mettre en pratique, cette activité, les stockeurs sont membres de groupements cotonniers dont les fonds servent à l'achat des céréales. A la récolte, le groupement de producteurs de coton achète une partie de la production de sorgho à ses membres. Les sacs sont stockés nominativement dans un même local en attendant que les prix augmentent. Le " grenier commun " est alors ouvert par le groupement à la 
demande des stockeurs. Afin d'intéresser les différents types d'exploitation, deux options se présentent :

- si le producteur dispose d'un stock alimentaire suffisant pour sa propre consommation jusqu'à la prochaine récolte, il ordonne au groupement de vendre son stock sur le marché à des prix plus élevés; le groupement se rembourse de l'avance qu'il a concédée et remet la marge bénéficiaire au stockeur ;

- si le producteur a épuisé son disponible alimentaire, il a la possibilité de racheter le sorgho à prix coûtant pour sa consommation.

Ainsi, ce système contribue à limiter les effets nocifs de l'endettement en favorisant une épargne en nature qui permet un recours modéré aux crédits usuraires. A plus long terme, les exploitations ont la possibilité de consolider leur gestion en reproduisant chaque année cette épargne en nature. Mais, il faut signaler que les résultats de ces activités sont mitigés, tant la gestion des magasins est conditionnée au recouvrement des céréales distribuées à crédit, ce qui s'avère illusoire dans les zones vulnérables. Face à la contrainte essentielle du renouvellement des fonds de roulement par le recouvrement intégral des crédits, la plupart des opérations de stockage communautaire n'ont pas trouvé de solutions appropriées et ont périclité. Les groupements formés pour ces opérations de stockage ont disparu avec les fonds destinés à l'achat de céréales. Enfin, le stockage des céréales fait face à plusieurs contraintes qui limitent leur efficacité. La contrainte principale réside dans l'insuffisance de ressources financières des groupements coton : seulement $25 \%$ des membres de ces groupements ont accès à ce stockage autofinancé et une partie importante de la production céréalière reste commercialisée à la récolte. A cela s'ajoute le manque de magasins qui empêche certains groupements de constituer un grenier commun (Abdourahmane et al, 2002).

Renforcer la représentation des producteurs céréaliers dans la filière : Les groupements coton comptent parmi les facteurs de réussite des greniers communs, notamment grâce à l'épargne collective disponible pour le financement des stocks de céréales. De plus, le suivi de la gestion des comptes par l'OPCCGIE est une garantie certaine pour le recouvrement des fonds. Cependant, les fonctions principales des groupements cotonniers relèvent plus de la production et de la commercialisation du coton que de la sécurité alimentaire. Une intervention est à envisager sur l'ensemble des filières céréalières, afin de généraliser les Greniers communs et de promouvoir une recherche-développement en agronomie pour lever certaines contraintes à la production et soutenir la diffusion des innovations par des organisations de producteurs. Afin d'affirmer leur place dans l'organisation de la filière céréalière, les organisations de producteurs doivent tenir un rôle prépondérant dans la conception et le suivi de projets en participant à leur maîtrise d'ouvrage. Pour éviter une rupture de la dynamique en cours, un programme d'appui à la filière apparaît indispensable, tant en aval pour une meilleure maîtrise des marchés des céréales par les producteurs, qu'en amont, pour améliorer les capacités de production. II serait donc plus utile de mettre en place d'un fonds de crédit auquel les banques de céréales peuvent faire appel pour financer leurs approvisionnements (avec un remboursement en fin de campagne de commercialisation).

Subventionner les intrants agricoles pour favoriser la production céréalière : Les défis et les enjeux d'amélioration de la productivité se posent également par rapport à l'approvisionnement en intrants. De lourdes hypothèques continuent de peser sur la qualité des intrants, la régularité de l'approvisionnement et l'efficacité des mécanismes de distribution sur lesquels se prononcent de plus en plus les producteurs regroupés au sein des coopératives, des groupements de producteurs, voir des interprofessions. Face aux coûts élevés d'accès aux intrants agricoles au cours de ces dernières années en zone cotonnière, il est utile de subventionner les intrants par l'Etat, comme cela s'amorce timidement par le ministère de l'agriculture depuis 2008, pour réduire le prix de vente aux producteurs afin de faciliter leur accès et les encourager à respecter les doses recommandées par la recherche. II attention particulière doit être accordée aux intrants vivrières indispensables pour la production céréalière afin de renforcer la sécurité alimentaire. Nous pensons aussi que l'Etat devra trouver des mesures incitatives pour encourager les operateurs économiques à s'intéresser à l'approvisionnement des intrants céréales dans la région.

Céréales pour la ville : Couvrir les besoins alimentaires des populations implique que l'offre soit en phase avec l'évolution des systèmes alimentaires. L'urbanisation induit un changement des modes de vie et impose la fourniture de produits transformés, plus faciles à préparer (temps de préparation, faible consommation d'énergie). La majorité des céréales mises sur le marché aujourd'hui le sont encore à l'état brut. Des initiatives de transformation des produits 
céréaliers (farines, couscous, etc.) se développent mais sont peu relayées par les politiques publiques. Le développement de l'artisanat alimentaire et la

\section{CONCLUSION}

En zone cotonnière du Cameroun la sécurité alimentaire reste précaire. Le coton a favorisé la culture céréalière à travers la relation étroite coton/céréales pendant de nombreuses décennies. Mais, depuis les années 1990, la crise de la filière cotonnière affecte aussi la production céréalière et donc la sécurité alimentaire déjà très précaire. En effet, avec la crise, l'accès aux intrants vivriers reste limité du fait des conditions d'accès liées à la culture du coton et à son coût de plus en plus élevé, mais aussi parce que les nombreux paysans qui abandonnent ou sont exclus de la culture cotonnière n'ont plus accès aux intrants vivriers à crédits et par conséquent, ils produisent sans engrais. Cette situation impacte les rendements et la production céréalière et par conséquent réduit l'offre en

\section{REMERCIEMENTS}

$\mathrm{Au}$ terme de cette analyse, nous tenons vivement à remercier le projet d'Appui à la Recherche Régionale pour le Développement Durable de Savanes d'Afrique Centrale (ARDESAC) pour les moyens financiers et

\section{REFERENCES}

Abdourahmane N, Mathieu B, Teyssier A, Bello R, 2002. La sécurité alimentaire : une affaire de paysans. In Jamin JY, Seiny Boukar L., Floret C. (éditeurs scientifiques), 2003. Savanes africaines : des espaces en mutation, des acteurs face à de nouveaux défis. Actes du colloque, mai 2002, Garoua, Cameroun. PRASAC, N'Djamena, Tchad - CIRAD, Montpellier, France. $10 \mathrm{p}$.

Badiane O, Ghura D, Goreux L, Masson P, 2002. "Cotton Sector Strategies in West and Central Africa", World Bank Policy Research Working Paper 2867, July 2002.

Banque Mondiale, 1986. La pauvreté et la faim : La sécurité alimentaire dans les pays en développement. Problèmes et options. Washington DC, Banque Mondiale. 1986.

Berti F, Hofs JL, Zagbaï HS, Lebailly P, 2006. Le coton dans le monde, place du coton africain et principaux enjeux. Biotechnol. Agron. Soc. Enviro (BASE), 10 (4) 271-280.

Dembélé NN, Staatz JM, 2010. Sécurité alimentaire en Afrique de l'Ouest : Enjeux et Eléments revalorisation de l'image des produits locaux constituent des enjeux majeurs pour les prochaines années.

céréale dans la région. Face à la crise, les producteurs tentent de s'adapter en réorientant les activités agricoles vers les vivriers, même si les superficies restent faibles, mais, ces cultures restent dépendantes de la culture du coton. Ces producteurs sont difficilement autosuffisants, bien que des tentatives de stockage des céréales soient effectuées, mais leurs actions restent très localisées et limitées face aux contraintes multiples. Enfin, afin de contribuer au renforcement de la sécurité alimentaire malgré la crise cotonnière. Des actions fortes doivent être menées, à savoir : le renforcement de la représentation des producteurs céréaliers dans la filière et la subvention des intrants agricoles pour favoriser la production céréalière entre autres.

logistiques mis à notre disposition pour réaliser ce travail. Enfin nos remerciements vont également à tous ceux qui ont contribué de près ou de loin à la réalisation de cette étude.

Stratégique pour sa Réalisation. MSU, Syngenta, USAID. $26 \mathrm{p}$.

Devèze JC, 2006. Le coton, moteur du développement et facteur de stabilité du Cameroun du Nord? Afrique contemporaine. $\mathrm{N}^{\circ} 217$ 2006/1, 107 à 120.

Djonnewa A, Kenga R, Obale EF, Ndikawa R, Njomaha C, 1997. Point de la recherche sur le sorgho de contre-saison au Nord-Cameroun. In Seiny Boukar L., Poulain J-F., Faure G., (eds), Agricultures des savanes du Nord-Cameroun : vers un développement solidaire des savanes d'Afrique centrale. Actes atelier d'échanges, 96, Garoua, Cameroun, Montpellier, France, CIRAD, p. 297-302

FAO, 2006. État de l'insécurité alimentaire dans le monde, Organisation des Nations Unies, Rome, 2006.

FAO, 2008. État de l'insécurité alimentaire dans le monde, Organisation des Nations Unies, Rome, 2008.

Fok ACM, 2010. Facteurs d'efficacité des arrangements institutionnels en politique 
cotonnière africaine. Cahiers d'Agricultures, Volume 19, $n^{\circ}$ 1. janvier-février 2010. 68 - 74.

Fofiri NEJ, Ndame JP, Temple L, Ndjouenkeu R, Simeu $\mathrm{KM}, 2008$. Croissance urbaine et innovations dans les filières vivrières : cas du maïs et du niébé dans les savanes du Cameroun, Ile Journées INRA-SFER-Cirad de recherches en sciences sociales, 11-12 décembre 2008, Lille, France.

Folefack DP, 2010. Coordination des acteurs dans un contexte de crise : le cas de la filière coton au Cameroun depuis 1990. Thèse de Doctorat en économie, Université Rennes 2 Haute Bretagne, Rennes, France, $333 \mathrm{p}$.

Folefack DP, Klassou C, Enam J, 2009. Price adjustments to cotton crisis in Cameroon: Factors and consequences of farmers' adaptations. Life Sciences International Journal, Special edition on cotton policies. 8999.

Institut National de la Statistique (INS), 2008. Troisième enquête camerounaise auprès des ménages (ECAM3) : Tendances, profil et déterminants de la pauvreté au Cameroun entre 2001-2007. Yaoundé, Cameroun. 51p.

Mana B, Wey J, Folefack DP, Havard M, 2009. Adaptation des assolements des exploitations agricoles du Nord Cameroun à la crise cotonnière. Colloque international " Savanes africaines en développement : Innover pour durer " 20 au 24 avril 2009, Garoua, Cameroun.

Mbetid-Bessane E, Havard M, Djondang K, 2006. Évolution des pratiques de gestion dans les exploitations agricoles familiales des savanes cotonnières d'Afrique centrale. Cahiers Agricultures $15, n^{\circ} 6: 555-561$

Mérino M, 2009. L'insécurité alimentaire en Afrique subsaharienne. Note de la Fondation pour la Recherche Stratégique, $n^{\circ}$ 01/2009.

MINEPAT, 2008. Autosuffisance et sécurité alimentaires au Cameroun : Une analyse basée sur la flambée des prix des Produits alimentaires de première nécessité. MINEPAT, Comite de pilotage du développement rural. Yaoundé, Cameroun. $55 \mathrm{p}$.

PAM, 2011. Analyse Globale de la Sécurité Alimentaire et de la Vulnérabilité au Cameroun. VAM, PAM, FAO Cameroun. $59 \mathrm{p}$.

SODECOTON, 2008. Note semestrielle d'information campagne 2007/2008, Juin 2008, 86 p.
Temple L, Fofiri NE, Ndame JP, Ndjouenkeu R, 2009. Impacts de la croissance urbaine sur l'innovation dans les filières vivrières du Nord Cameroun. In L. Seiny-Boukar, P. Boumard (éditeurs scientifiques), 2010. Actes $d u$ colloque "Savanes africaines en développement : innover pour durer ॥, 20-23 avril 2009, Garoua, Cameroun. PRASAC, N'Djaména, Tchad ; CIRAD, Montpellier, France, cédérom.

Tschirley D, Poulton C, Gergely N, Labaste P, Baffes J, Boughton D, Estur G, 2009. Méthode d'analyse des effets différentiels des réformes sur les filières cotonnières en Afrique. Cahiers d'Agricultures, vol. 18, $n^{\circ}$ 5, 385-392. 